\title{
La investigación sobre ciudad y comunicación: un
}

campo abierto*

\author{
The research on city and communication: an open field
}

anna Clua

Universitat Oberta de Catalunya (Estado español)

\section{Resumen}

El presente texto apuesta por una visión interdisciplinar y comprensiva de las relaciones entre ciudad y comunicación. Se hace necesario interpretarlo, por ello, desde una mirada abierta y colaborativa para con las distintas disciplinas académicas que concurren a definir tales vínculos, sus prácticas y sus resultados.

La autora señala cuáles pueden ser los diferentes marcos conceptuales de esta relación trazando un primer mapa orientativo desde el que emerge la Comunicación y en el que se caracteriza su permeabilidad.

Así, se ofrece una reflexión sobre la configuarción epistemológica del campo, que permite ubicar las líneas de investigación que abrieron camino y, en un segundo apartado, se presentan diez conceptos relevantes que se han fabricado o reconvertido desde la academia iberoamericana desde finales del siglo veinte hasta inicios del veintiuno.

\section{Abstract}

This text stands up for an interdiciplinary and comprehensive vision of the relationships between the city and communication. Therefore, it is necessary to start from an open and collaborative point of view on the different academic disciplines from where the practices and the results of these liaisons could be defined.

The author points to several conceptual frames that can be listed, and develops a preliminary map where Communication emerges as a permeable space.

Thus, she provides an epistemological reflection on the setting up of this field, which allows locating the opening research areas, as well as identifying ten relevant concepts that have been generated or adopted by the Ibero-American academy by the end of the 20th century and the early 21 st century.

\section{Palabras clave}

Ciudad, comunicación, epistemologías, investigación, iberoamérica

\section{Keywords}

City, communication, epistemologies, research, Iberoamerica

\footnotetext{
* El texto recuperado y revisado para Revistero fue publicado como Clua, A. (2010). La investigación sobre ciudad y comunicación: un campo abierto, En M. Martínez Hermida, (Coord.) Ciudad y Comunicación (pp. 11-25). Madrid: Fragua.
} 


\section{Sumario}

1. Introducción

2. El debate epistemológico en la investigación sobre ciudad y comunicación

3. Diez formas de abordar la relación entre ciudad y comunicación

3.1. Comunicación y configuración histórica de la ciudad

3.2. Comunicación y construcción de identidades culturales urbanas

3.3. Comunicación y desarrollo urbano

3.4. Medios comunitarios como fenómeno urbano

3.5. Ciudad y periodismo

3.6. La ciudad de los arquitectos

3.7. Ciudad informacional

3.8. Ciudad del conocimiento

3.9. Ciudad virtual/ cibernética

3.10. Ciudad espectáculo y marketing urbano

4. Reflexión final

\section{Contents}

1. Introduction

2. The epistemological debate in the field of city and communication

3 . Ten ways of tackling the relationship between city and communication

3.1. Communication and historical configuration of the city

3.2. Communication and construction of urban identities

3.3. Communication and urban development

3.4. Community media as an urban phenomena

3.5. City and journalism

3.6. The city of architects

3.7. The information city

3.8. City of knowledge

3.9. Virtual and cyber city

3.10. City of the spectacle and urban marketing

4. Final comments 


\section{INTRODUCCIÓN}

La relación entre ciudad y comunicación ha sido objeto de interés en numerosos estudios hasta el día de hoy. Por un lado, se ha analizado el fenómeno urbano desde el campo de la comunicación. Por otro lado, ha sido el fenómeno de la comunicación en la ciudad lo que ha centrado la atención de estudiosos provenientes de campos como la geografía urbana, la economía, las ciencias políticas, el urbanismo o la arquitectura.

Para las y los investigadores que venimos del campo de la comunicación los primeros trabajos sobre la ciudad se sitúan en el marco de la ecología urbana desarrollada desde la Escuela de Chicago, a principios del siglo $X X$, en un contexto profundamente marcado por la ciencia positivista. Desde entonces, la aproximación al fenómeno urbano ha experimentado numerosos cambios en cuanto al planteamiento epistemológico se refiere. En gran medida, el estudio de la ciudad y la comunicación ha evolucionado de forma paralela a las transformaciones de nuestras sociedades (es decir, no puede explicarse al margen de ellas).

Cabe mencionar aquí la existencia de textos donde, desde el campo de estudio de la comunicación, ya se ha reflexionado acerca de la investigación sobre ciudad y comunicación. En el contexto iberoamericano destacan los trabajos de Gómez-Mompart (1996), Reguillo (1997) o Badenes (2007).

En el presente texto se parte de la idea de que la complejidad del fenómeno de la comunicación en la ciudad, junto con la creciente velocidad de los cambios que se producen en la actualidad, hace necesaria una visión interdisciplinar y comprensiva del objeto de estudio. Es decir, en lugar de reivindicar una aproximación al fenómeno desde un "campo" determinado (como pudiera ser el de la comunicación), se subrayará la necesidad de adoptar una mirada más abierta, y una práctica científica más colaborativa con otras disciplinas académicas.

El primer paso hacia el reconocimiento de este "campo abierto" de estudio es la identificación de los diferentes marcos conceptuales desde donde se puede abordar el estudio de la comunicación en la ciudad. Las páginas que siguen van a dedicarse enteramente a dibujar este mapa orientativo. Con ello se pretende ampliar el marco de referencia que hasta aho- ra ha definido el estudio de la ciudad desde el ámbito de la comunicación. No se trata tanto de negar que exista "un" campo de estudio sobre el fenómeno de la comunicación en los procesos urbanos, sino más bien de apostar por una mayor permeabilidad del mismo.

Las páginas que siguen proponen, en primer lugar, una reflexión sobre la evolución epistemológica en el marco de las ciencias sociales donde debemos situar las distintas líneas de investigación. En un segundo apartado se presentarán diez conceptos utilizados en los estudios sobre ciudad y comunicación que se han desarrollado en Iberoamérica, intentando limitar esta descripción a nuestro contexto académico más inmediato (y evitando reproducir aquí la tendencia a tomar como únicos referentes a los trabajos desarrollados en el contexto anglosajón). El texto concluirá con una reflexión crítica sobre el vasto "campo" que se abre alrededor del estudio de la ciudad y la comunicación.

\section{EL DEBATE EPISTEMOLÓGICO EN LA INVESTIGACIÓN SOBRE CIUDAD Y COMUNICACIÓN}

Tanto la ciudad como la comunicación son temas de estudio complejos en tanto que multidimensionales. La relación entre ambos temas ha sido investigada de muy distintas maneras (bien considerando la comunicación como factor colateral, bien como elemento vertebrador), dependiendo del enfoque disciplinar de las investigaciones (Gómez-Mompart, 1999). Existen, sin embargo, ciertos paralelismos entre estas diversas perspectivas por lo que se refiere a su evolución epistemológica, cosa que es más fácilmente detectable si situamos nuestra mirada en el marco de las ciencias sociales en general.

En este sentido, vemos cómo las distintas investigaciones (independientemente de su etiqueta disciplinar) evolucionan en base a la aceptación o refutación de los paradigmas de conocimiento. Un primer cambio epistemológico se produce en el momento (durante la primera mitad del siglo $\mathrm{XX}$ ) en que empieza a cuestionarse la hegemonía del positivismo en las ciencias sociales, aunque aquí el cambio no implica todavía una negación del pensamiento paradigmático (como demuestra la evolución de perspectivas críticas como, por ejemplo, la Escuela de Frankfurt). Al mismo tiempo, surgen las perspectivas académicas 
que cuestionan el modelo de objetividad científica que presupone una postura a-política por parte del investigador. Es la época, pues, de la irrupción de las corrientes marxistas en las ciencias sociales.

El segundo momento de cambio epistemológico se situaría ante la aparición de perspectivas distintas a las instauradas por las teorías funcionalistas (lo que no es lo mismo que afirmar que estas últimas no continúen ejerciendo una gran influencia en la actualidad), así como ante el salto de las metodologías cuantitativas a las metodologías cualitativas. Este cambio atenta mucho más directamente contra el tipo de conocimiento que se erige bajo la "etiqueta" de un determinado paradigma, afectando en gran medida la forma de definir el objeto de estudio.

Este es el momento (a partir de la segunda mitad del siglo XX) en que podemos situar, por ejemplo, el "giro cultural" que se experimenta en el seno de las ciencias sociales, y que en cierto modo empieza a acercar ámbitos académicos tradicionalmente distanciados (por ejemplo, la economía política y los estudios culturales). En muchos casos, el posicionamiento político de los investigadores hace también un giro hacia una nueva izquierda más alejada del marxismo ortodoxo/dogmático/determinista y que intenta recuperar conceptos que dan pie a incluir a la cultura en la reflexión sobre las relaciones de dominación/transformación de la sociedad (como, por ejemplo, los conceptos gramscianos de "hegemonía" o de "intelectualidad orgánica").

En el caso específico de los estudios sobre comunicación, todos estos cambios implican (entre otras cosas) el abandono de posturas mediacéntricas (Martín-Barbero, 1987) para ubicar el objeto de estudio en la sociedad misma y en el tipo de relaciones que se dan entre los distintos agentes que la configuran. Aumenta así el interés por comprender históricamente el fenómeno comunicativo en tanto que eje vertebrador de la configuración/transformación de las identidades culturales. Con este planteamiento se cuestiona, pues, que la comunicación deba describirse como un proceso lineal y unidireccional, canalizado únicamente por los medios de comunicación masivos y dirigido a un público amorfo y pasivo.

Hay autores que defienden la idea de que en la actualidad estamos ante un tercer momento de cambio epistemológico. Éste es el producido por la revolución tecnológica y por la transformación social que supone el paso de la era industrial a la era de la información (Castells, 1995; 1997). Así, los anteriores modelos de pensamiento dan paso a un acercamiento a los fenómenos sociales concebidos como una red global de acontecimientos (y no tanto como una consecución de causas y efectos, o como fenómenos situados en un punto concreto de la geografía y/o de la historia).

El tipo de conocimiento generado en la era de la información es, pues, el marco de referencia que toman muchas de las actuales investigaciones sobre la comunicación y la ciudad (por ejemplo, desarrollando temas de estudio como el de las "ciudades del conocimiento", las "ciberciudades", las "smart cities", etc.). No obstante, merece la pena apuntar aquí la duda de si la presencia cada vez más hegemónica de este enfoque ciertamente innovador (en las agendas de investigación o en las prioridades editoriales de las publicaciones académicas) no supone a menudo una regresión hacia posturas epistemológicas poco comprometidas con la idea de una transformación real de los esquemas de pensamiento. En todo caso, desde el presente texto no se obviará el hecho de que esta perspectiva haya recibido críticas en tanto que falsa revolución del conocimiento (Mattelart, 2007). En lo que afecta al estudio de la comunicación se ha criticado, por ejemplo, que esta línea de trabajo reproduzca un "tecnocentrismo" difícil de desvincular de aquella tradición funcionalista que se pretendía en entredicho.

\section{DIEZ FORMAS DE ABORDAR LA RELACIÓN ENTRE COMUNIDAD Y COMUNICACIÓN}

La investigación sobre ciudad y comunicación ha generado distintas formas de nombrar a su objeto de estudio. Los diez conceptos que van a presentarse a continuación servirán para dar cuenta de la diversidad de planteamientos epistemológicos de los que se ha hablado en el apartado anterior. Con ello no se pretende construir un estado de la cuestión que comprenda un listado exhaustivo de temas, autores u obras publicadas. El objetivo de este apartado es simplemente ofrecer un ejemplo ilustrativo del tipo de líneas de trabajo que han tenido una mayor incidencia en el contexto iberoamericano. 
Se han escogido diez conceptos o ideas a partir de los cuales se puede describir el binomio ciudad/comunicación. Su presentación se realizará bajo la forma de resumen breve, con referencias a textos y autores representativos de diferentes posturas epistemológicas. Como se ha dicho antes, los trabajos referenciados en cada uno de los sub-apartados no deben ser considerados como los únicos existentes, sino como meros ejemplos del tipo de textos publicados en nuestro contexto académico más inmediato. Queda abierta, pues, la puerta para futuras profundizaciones sobre el tema.

Las diez formas de abordar la relación entre ciudad y comunicación a las que se hará mención aquí son las siguientes:

- Comunicación y configuración histórica

de la ciudad

- Comunicación y construcción de

identidades culturales urbanas

- Comunicación y desarrollo urbano

- Medios comunitarios como fenómeno urbano

- Ciudad y periodismo

- La ciudad de los arquitectos

- Ciudad informacional

- Ciudad del conocimiento

- Ciudad virtual / ciberciudad

- Ciudad espectáculo y marketing urbano.

\subsection{COMUNICACIÓN Y CONFIGURACIÓN HISTÓRICA DE LA CIUDAD}

A finales de la década de 1980 el libro De los medios a las mediaciones, de Jesús Martín-Barbero, supuso (entre otras cosas) un primer intento de explicar cómo la radio y el cine contribuyeron a construir la imagen de "modernidad" en Latinoaérica (Martín-Barbero, 1987). En ese mismo período aparece en España el interés por el tema, desarrollándose una serie de trabajos desde la perspectiva de la historia de la comunicación y aplicados al estudio de las ciudades. Concretamente, estos trabajos proponen un análisis histórico del fenómeno de los medios de comunicación de masas en la era moderna, centrándose en el papel de los mass media en la configuración de la "sociedad-cultura de comunicación de masas", así como en el papel de las ciuda- des como enclaves de la modernidad (GómezMompart, Marín y Tresserras, 1989; GómezMompart, 1992; Espinet, 1993).

\subsection{COMUNICACIÓN Y CONSTRUCCIÓN DE IDENTIDADES CULTURALES URBANAS}

El análisis del papel de los medios de comunicación en la formación de identidades culturales urbanas constituye una de las líneas de investigación con mayor proyección y continuidad en el tiempo. Los estudios iniciaron su andadura a finales de los años 80 en Latinoamérica, centrándose en el análisis crítico del conflicto entre la forma urbanística de la ciudad y la representación de los imaginarios sociales (ciudades vividas, ciudades percibidas, ciudades deseadas, ciudades cotidianas, ciudades planificadas...). En esta línea de investigación confluyen perspectivas como los estudios culturales, los estudios de comunicación o la antropología. Destacan los trabajos de Silva (1988), Martín-Barbero (1994), Lombardi (1995), Reguillo (1996) o García Canclini (1999).

\subsection{COMUNICACIÓN Y DESARROLLO URBANO}

De Latinoamérica proviene también la propuesta de estudiar la relación entre comunicación y desarrollo urbano. Este tema se viene desarrollando desde principios de los años 90 con un enfoque interdisciplinar, pero con liderazgo de los estudios sobre comunicación. El origen de esta línea de investigación se sitúa alrededor de los Programas de la UNESCO en América Latina (Oficina Regional de Comunicación). Estos programas dieron pie al desarrollo de proyectos de investigación en varias universidades ${ }^{1}$. Desde esta perspectiva se insiste en la necesidad de dejar de pensar la ciudad como el "ámbito donde se observan algunos fenómenos de comunicación" para pensar "la ciudad desde la comunicación" (Miralles, 2001). También se abandona el paradigma desarrollista dominante anglosajón. En este tipo de proyectos el "desarrollo urbano" se define como la lucha contra la exclusión, o como la rehabilitación del espacio público, el fomento de la participación ciudadana o la

${ }^{1}$ Pontificia Bolivariana de Medellín; Javeriana de Bogotá, Católica de Montevideo, Metodista de Sao Paulo, Iberoamericana de México, Diego Portales de Chile, Universidad de Lima. 
libre información. La investigación se desarroIla entorno a cuatro ejes principales de traba. a. La crisis de comunicación en la ciudad b. La comunicación en la planificación urbana c. El papel de los medios de comunicación en la ciudad

d. La información y la comunicación administrativa

Actualmente en España se están realizando trabajos en esta misma línea y en colaboración con Latinoamérica (Sierra, 2006).

\subsection{MEDIOS COMUNITARIOS COMO FENÓMENO URBANO}

El análisis de los medios comunitarios como fenómeno urbano constituye un cuarto enfoque de los estudios sobre ciudad y comunicación. Si bien los medios comunitarios empezaron a ser analizados ya en la década de los 60 (en muchos casos, bajo la influencia de la obra de Paulo Freire), no es hasta los años 80 que este tema de investigación produce un importante número de publicaciones desde una gran diversidad de enfoques académicos. Destacan, por ejemplo, los trabajos realizados desde el campo de los estudios de comunicación, la sociología de los movimientos sociales, los estudios culturales, el enfoque del derecho constitucional, los estudios sobre regeneración urbana, o bien los trabajos en pedagogía.

Este tipo de estudios parte de una clara vocación empírica de la investigación, dando pie a un enfoque que partiendo del concepto de la acción comunicativa (en tanto que forma de participación política) desemboca, ya en la década de los años 2000, en una defensa del activismo desde la academia. La ciudad, en este sentido, es el marco donde preferentemente tiene lugar esta "apropiación ciudadana de los medios" (Winocur, 2002; Crovi, 2003; Chaparro, 2004; López Cantos, 2004; Mayugo, 2005).

\subsection{CIUDAD Y PERIODISMO}

Los estudios sobre ciudad y periodismo han sido abundantes desde hace muchas décadas. A menudo, el relato periodístico sobre la ciudad ha sido abordado en tanto que relato literario (sobre todo por lo que respecta a la prensa de finales del XIX o principios del XX). Otras veces, ya entrada la década de 1970, la ciudad de los periodistas se configuró como un nuevo espacio de acción política coincidiendo, por ejemplo en España, con la joven reinstauración de la democracia en los municipios, así como con la transformación urbana reivindicada desde las periferias proletarias. La ciudad así vista por los periodistas ocupó varios volúmenes de libros y numerosos artículos en publicaciones especializadas (Fabre y Huertas, 1976; Huertas y Vilasaró, 1982).

Enlazando con esta última perspectiva, a partir de la década de los 80 y, sobre todo, en los años 90 aparece con fuerza en el contexto académico iberoamericano el análisis del periodismo urbano como forma de "periodismo ciudadano". En cierto modo, esta corriente pretende romper con la tradición del civic journalism desarrollado en Estados Unidos, en un intento de traer a una realidad más cercana en el espacio/tiempo la decana reflexión teórica sobre el papel del periodismo en las sociedades democráticas, la formación de la opinión pública o la transformación del espacio público. Así, aparecen en las publicaciones académicas del contexto iberoamericano conceptos como el de "periodismo público", así como una mayor atención hacia la relación entre comunicación y ciudadanía (Dader, 1992; Correia, 1998, Miralles, 2002; Traquina y Mesquita, 2003). Se percibe también un acercamiento de esta reflexión a la desarrollada paralelamente (y contemporáneamente) alrededor de los medios de comunicación comunitarios, aunque sin converger plenamente con ella.

\subsection{LA CIUDAD DE LOS ARQUITECTOS}

Algo antes que la "ciudad de los periodistas" nació, ya en la década de 1960, una "ciudad de los arquitectos" analizada también desde un punto de vista "orgánico". Se trataba de la ciudad concebida por una "nueva arquitectura" que intentaba subrayar el carácter social del arte y, concretamente, de las artes aplicadas a la transformación de la ciudad. Los jóvenes arquitectos de la época reivindicaban así un "método polémico" con el que crear y comunicar espacios urbanos "sociales", y que implicase una mayor reflexión sobre la relación entre la realidad física y la cultural, así como una mayor conciencia crítica del papel de la arquitectura en la construcción de la ciudad. Desde este punto de vista, se hacía un llamamiento para que los arquitectos no se convirtieran en "arbitrarios 
dictadores" de un urbanismo exclusivamente técnico, sino técnicos al servicio de la sociedad (Bohigas, 1963).

El lenguaje arquitectónico se convierte así en una forma de comunicar la metamorfosis urbana. Esta comunicación no va dirigida a un grupo de gente privilegiada en tanto que capaz de decodificar el mensaje de la arquitectura. Lo que se pretende es precisamente socializar el significado del espacio. Ya no se trata sólo de crear espacios funcionales por encargo de quien ostenta el poder, sino de dotar a la ciudadanía de espacios públicos (físicos y simbólicos) donde poder expresarse libremente. En España este fenómeno cobró especial importancia en la época de declive del régimen dictatorial de Franco. Curiosamente, el análisis posterior de la labor realizada por esta "nueva arquitectura" corrió a cargo de analistas ajenos a este ámbito. En este sentido, resulta especialmente ilustrativo el trabajo del periodista Llàtzer Moix sobre la transformación urbana como "acontecimiento cultural" de la Barcelona de los años de transición política hacia la democracia (Moix, 1994).

\subsection{CIUDAD INFORMACIONAL}

En el contexto iberoamericano, la ciudad de la Era de la Información empieza a ser objeto de análisis sobre todo a partir de finales de la década de 1980. Conceptos como el de ciudad-nodo de comunicación, ciudad-región, ciudad global, ciudad-red o red de ciudades surgen con fuerza a partir de los estudios desarrollados en el campo de la geografía urbana, la economía política de la ciudad o la sociología (Castells 1995; Borja y Castells, 1997).

El centro de atención de la perspectiva de la ciudad informacional es, pues, el de la transformación del papel de la ciudad en el contexto de la economía globalizada (una economía post-fordista, mucho más basada en el mundo financiero que en el de la producción, y que supone la evolución de los enclaves urbanos hacia una economía del conocimiento, así como una mayor movilidad tecnológica, humana y de capital a escala mundial). Atrás parece quedar, pues, la ciudad industrial y los procesos culturales de formación de identidad que le eran atribuidos.

Esta perspectiva de análisis incide sobre todo en el tema de la transformación de los procesos urbanos a partir de la llegada de las nuevas tecnologías de la información y la comunicación. Por ejemplo, se observa cómo las TIC afectan la gestión urbana, tanto por lo que se refiere a nuevas formas de gobierno como a nuevas formas de gobernanza. Este último punto ha sido tratado desde los estudios sobre e-governance, analizando el impacto de las nuevas tecnologías en la administración pública (Castells y Ollé, 2004; Finquelievich et al., 2004).

\section{8. CIUDAD DEL CONOCIMIENTO}

Estrechamente ligado al concepto de ciudad informacional surge, a partir de los estudios desarrollados en los años 1990, el concepto de ciudad del conocimiento. La relativa autonomía de este concepto respecto del anterior radica en su capacidad de haber generado un prolífico corpus de trabajos y publicaciones bajo ese epígrafe. Conviene destacar aquí la gran influencia ejercida por las investigaciones llevadas a cabo en el ámbito anglosajón. Así, las knowledge-based cities se erigen como un nuevo concepto de interés académico, con un gran potencial por su elevado índice de impacto tanto en las agendas de investigación como en el diseño de políticas municipales. Los conceptos-clave que caracterizan esta línea de trabajo están estrechamente vinculados al tipo de lenguaje utilizado actualmente en ámbitos económicos. Destacan, por ejemplo, los estudios sobre "nuevo emprendedurismo", "industrias creativas", "innovación", “distritos tecnológicos", etc.

Las perspectivas de análisis desarrolladas alrededor de la ciudad del conocimiento responden a diversas posturas epistemológicas, y sólo recientemente han admitido un cierto mestizaje con disciplinas distintas a la economía o el urbanismo. Así, por un lado, se han llevado a cabo estudios sobre el desarrollo de la ciudad como capital de la innovación y como polo atrayente de actividades económicas ligadas al tipo de producción cultural que generan las nuevas formas de comunicación (Trullén et al., 2002). Por otro lado, existen trabajos que implican una crítica abierta a la ciudad del conocimiento como enclave de la privatización tanto de la gestión urbana como de los espacios de comunicación ciudadanos (Clua y Albet, 2008). 


\subsection{CIUDAD VIRTUAL/CIBERCIUDAD}

Los estudios sobre la ciberciudad empezaron a desarrollarse a partir de los años 90 y a raíz de la proliferación de programas informáticos de simulación. El análisis de este fenómeno se volcó mayoritariamente hacia el estudio del consumo de nuevas formas de ocio, como pueden ser los videojuegos de recreación virtual de la vida urbana. Este enfoque ha sido desarrollado también en relación al estudio de la formación de comunidades virtuales.

Las ciudades virtuales en Internet también han sido foco de interés de los estudios de marketing. En este sentido, las ciberciudades se han concebido como espacios de publicidad para la actividad comercial y turística de ciudades reales. La observación del acceso de los consumidores a estos "escaparates" comerciales en Internet permite explorar el alcance de fenómenos como la tele-compra o los centros comerciales virtuales.

El tema de la participación ciudadana también tiene cabida en los estudios sobre ciudades virtuales. En este sentido, no prima tanto el interés por el uso de programas de simulación o el acceso a Internet en tanto que actividad de consumo, sino que se prioriza el análisis de la participación activa de la ciudadanía a la hora de diseñar espacios urbanos.

El interés académico por todos estos temas se inició en el ámbito anglosajón, desde perspectivas como la de los estudios culturales, la geografía cultural, la arquitectura o la antropología. En el contexto iberoamericano existe un menor número de este tipo de estudios, y es sobre todo a partir de los últimos años 2000 cuando se publican algunos trabajos (López et al., 2006; Vivas et al., 2008).

\section{10. CIUDAD ESPECTÁCULO Y MÁRKETING URBANO}

Los estudios sobre la ciudad espectáculo empiezan su andadura en nuestro contexto académico hacia los años 80 . Desde el punto de vista de la comunicación, las perspectivas de análisis más destacadas se desarrollan principalmente alrededor de dos temas: la ciudad como discurso de los planificadores y urbanistas, y la ciudad como discurso de los anunciantes.

Los conceptos clave que se utilizan en este tipo de trabajos redundan en la idea de que la imagen de la ciudad es actualmente definida por la lógica de los anuncios publicitarios, expresándose a menudo a través del lenguaje audiovisual. Así, en los estudios aparecen términos como "ciudad espectáculo", "ciudad anuncio", "imagen de la ciudad" o "ciudad plató". Como trasfondo de esta "mirada" sobre el espacio urbano existe una crítica a la construcción simbólica de la ciudad en tanto que espacio de consumo (Benach, 2000). También se cuestiona la naturalización con la que se habla de las ciudades como "marca" de un mercado global urbano dinamizado por la competitividad (Fernández y Paz, 2005). Los estudios de geografía urbana juegan un papel destacado en este ámbito temático.

Por un lado, se cuestiona la sostenibilidad de un modelo urbano que fomenta la privatización de la experiencia de los ciudadanos en tanto que consumidores de la ciudad. Por otro lado, se critica la supeditación de la iniciativa pública a los intereses del capitalismo tardío hegemónico. En este sentido, se analiza la generalización del desarrollo urbano alrededor de grandes eventos que funcionan como fuente de atracción de inversiones internacionales (y por tanto, como formas de incrementar la competitividad de las ciudades en el contexto global). A través de olimpiadas, festivales, forums, rodajes de películas... las ciudades son convertidas en escenarios, cuando no en espectáculos propiamente (Sánchez, 1997; Benach y Albet, 2005; Fernández y Paz, 2005).

\section{REFLEXIÓN FINAL}

El punto de llegada de esta comunicación podría resumirse con la siguiente afirmación: La investigación sobre ciudad y comunicación es un campo abierto, no tanto porque sea un ámbito joven a medio construir, sino más bien porque no existe un punto de encuentro de todos los trabajos realizados hasta el momento. Cada perspectiva ha desarrollado su propio enfoque, y muy raramente se ha trabajado codo a codo con otros investigadores que abordan los mismos temas. No existe, pues, un campo de conocimiento del que surjan propuestas de análisis, sino varias perspectivas que preguntan por separado cuál es la relación entre ciudad y comunicación.

Este hecho no es considerado aquí como una debilidad o un problema. De entrada, se parte de la idea de que la diversidad de miradas no es algo negativo. Sí lo es, en cambio, la 
falta de diálogo entre diferentes perspectivas, así como la falta de debate crítico en el seno de la producción académica. Se ha visto, por ejemplo, como dentro del campo de estudio de la ciudad y la comunicación los avances de una perspectiva desarrollada desde hace décadas son raramente incorporados como punto de partida de los análisis de fenómenos recientes. Es decir, además de la dificultad de trascender barreras disciplinares a la hora de enfocar un objeto de estudio existe cierta división temporal de las investigaciones, como si de un avance del conocimiento "por modas" se tratara.

A fin de abrir un interrogante sobre el futuro de los estudios sobre ciudad y comunicación en el contexto iberoamericano se proponen tres puntos de reflexión. Con ello, se pretende vincular la descripción de lo que aquí se ha denominado un "campo abierto" con el tipo de limitaciones que caracterizan hoy en día la producción académica. A saber:

1. La propia estructura de la academia y las rutinas que de ella se derivan han dificultado el diálogo interdisciplinar. A pesar de la existencia de planteamientos epistemológicos proclives a romper con el estancamiento de la "territorialidad académica", en escasas ocasiones se han abierto espacios que se hayan consolidado como lugares de trabajo conjunto. La mayoría de experiencias en este sentido han constituido tan sólo lugares puntuales de intercambio entre disciplinas (proyectos de investigación con fecha de caducidad, seminarios temáticos en el seno de congresos, etc.). Es más: los escasos lugares de trabajo conjunto que se han consolidado han sido creados, generalmente, gracias a iniciativas externas a la propia academia (como organizaciones gubernamentales, instituciones públicas o privadas, etc.).

2. El distanciamiento entre distintas "culturas" académicas es un problema difícil de subsanar por una razón que aparentemente es idiomática pero que en realidad tiene muchas más connotaciones "geoestratégicas". Como en muchos otros campos de estudio, existe todavía un abismo entre los estudios en ciudad y comunicación que se llevan a cabo en el ámbito anglosajón y los que se desarrollan en el ámbito iberoamericano. Muchas veces, los proyectos que se inician en nuestro contexto más inmediato van a la zaga de los "avances" que sobre esta materia nos llegan desde el norte de Europa o Estados Unidos.
Ello supone que, salvo contadas excepciones, en lugar de consolidar conocimientos "situados" vayamos reproduciendo la tendencia a seguir las "modas" o las líneas de trabajo que se dictan desde países donde la investigación cuenta con mejores condiciones de desarrollo y difusión.

3. Los estudios sobre ciudad y comunicación que se están desarrollando "sobre la marcha" atienden a las necesidades del momento más que a los objetivos a largo plazo que acompañan el desarrollo de un campo de conocimiento. En este sentido cabe destacar la falta de protagonismo y de independencia de los científicos sociales a la hora de diseñar su agenda de trabajo. No estamos hablando de la investigación funcionalista de antaño, sino de las prácticas que hoy en día marcan las rutinas académicas. En un contexto cada vez más competitivo donde prima la máxima productividad a corto plazo y la obtención de resultados inmediatos, no es de extrañar que los estudios adapten sus objetivos a la necesidad primordial de obtener financiación o reconocimiento curricular individual. Ello tiene consecuencias tanto en lo que se refiere a las prioridades de estudio como en la puesta en práctica de una conciencia crítica respecto a las necesidades sociales de conocimiento.

Está claro que no todas las formas de estudiar la relación entre ciudad y comunicación (tal y como se han presentado en el apartado 3) responden de igual modo a estas limitaciones. El presente texto ha intentado ofrecer una crítica a todo aquel desarrollo de los estudios sobre ciudad y comunicación que no haya sido capaz de combinar el análisis histórico con la comprensión de la realidad presente. Se critica también la fascinación producida por las tecnologías en tanto que motores únicos de innovación social y desarrollo urbano. El punto de vista secundado aquí es, pues, el de la apuesta por un análisis en mayor profundidad de la relación entre los procesos económicos y los procesos políticos/sociales/ culturales que caracterizan la evolución de las ciudades y de las formas de comunicación que se dan en ellas. Por último, el planteamiento de este texto ha pretendido cuestionar el uso de conceptos teóricos como espacios comunes, sin mediar ninguna crítica sobre el tipo de discurso o la mirada epistemológica que se reproducen al estudiar la comunicación en la ciudad y la ciudad en la comunicación. 


\section{$>$ Referencias Bibliográficas}

- Badenes, D. (2007). Comunicación y ciudad: líneas de investigación y encuentros con la historia cultural urbana. Question, 14, 1-14.

- Benach, N. (2000). Nuevos espacios de consumo y construcción de la imagne de la ciudad en la Barcelona olímpica. Estudios Geográficos, 61 (238), 189-205.

- Benach, N. y Albet, A. (2005). Barcelona 1979-2004, entre el modelo y el espectáculo. En Minca, C. (Ed.) Lo spettacolo della città/ The spectacle of the city (pp. 1-34). Padova: CEDAM

- Bohigas, O. (1963). Barcelona: entre el Pla Cerdà i el barraquisme. Barcelona: Edicions 62

- Borja, J. y Castells, M. (1997). Local y global. La gestión de las ciudades en la era de la información. Barcelona:

Taurus.

- Castells, M. (1995). La ciudad informacional. Tecnologías de la Información reestructuración económica y el proceso urbano-regional. Madrid: Alianza Editorial.

- Castells, M. (1997). La era de la Información. Vol I: La sociedad red. Madrid: Alianza Editorial.

- Castells, M. y Ollé, E. (2004). El model Barcelona II: L'Ajuntament de Barcelona a la societat xarxa de Catalunya (2002-2004). Projecte Internet Catalunya: Govern electrònic i administració pública. Barcelona: UOC.

- Chaparro, M. (2004). Mediacentro. La propuesta de comunicación participativa para las ciudades y los barrios de la red EMA RTV. En Marí, V. (coord.) La Red es de todos. Cuando los Movimientos Sociales se apropian de la Red (pp. 137153). Madrid: Editorial Popular.

- Clua, A. y Albet A. (2008). 22@bcn Plan: Bringing Barcelona Forward in the Information Era. En Yigitcanlar, T., Velibeyoglu , K.y Baum, S. (Eds.) Knowledge-Based Urban Planning and Applications in the Information Era. (pp. 132-147). Hershey, New York: IGI Global.

- Correia, J. C. (1998). Jornalismo e espaço público. Covilhã, Beira Interior: Serviços Gráficos da Universidade da Beira Interior.

- Crovi, D. (2003). Ciudadanizar la radio y la televisión. Que todas las voces se expresen. I Foro Los medios son de todos, diciembre 2003, Cuernavaca, Morelos: México.

- Dader, J. L. (1992). El periodista en el espacio público. Barcelona: Bosch cietat-cultura de comunicació de masses a Catalunya a través del egodocuments, 1888-1939, (Tesis doctoral), Bellaterra, Barcelona: UAB

- Fabre, J. y Huertas, J.M. (1976). Tots els barris de Barcelona. Barcelona: Edicions 62

- Fernández, G. y Paz, S. (2005). Más allá del marketing de ciudades: hacia una política pública de diseño y gestión de los signos de identificación de ciudad. Scripta Nova. Revista electrónica de geografía y ciencias sociales, vol. IX, 194 (94)

- Finquelievich, S., Lago, S., Jara, A. Baumann, P., Pérez, A. y Zamalvide, M (2004). The social impact of introducing ICTs in local government and public services: Case studies in Buenos Aires and Montevideo. En Bonilla, M. y Cliché, G. (Eds.) Internet and Society in Latin America and the Caribbean (pp. 147-192). Ontario: Southbound/IDRC Books

- García Canclini, N. (1999). Imaginarios Urbanos. Buenos Aires: Eudeba.

- Gómez-Mompart, J.L., Marín, E. y Tresserras, J.M. (1989). Premsa, comu nicació $i$ cultura a Catalunya durant el primer terç del segle XX. Bellaterra Barcelona: Facultat de Ciències de la Informació, UAB.

- Gómez Mompart, J. L. (1992). La gène si de la premsa de masses a Catalunya (1902-1923). Barcelona: Pòrtic.

- Gómez Mompart, J. L. (1996). Ciudades y comunicación en la periferia atlántica: ecosistemas comparados desde la perspectiva histórica. En actas de Comunicación na periferia atlántica (pp. 391-394). Santiago de Compostela: Facultade de Ciencias da Comunicación USC

- Gómez Mompart, J. L. (1999). La ciudad es el medio, el territorio es el mensaje. Comunicar, 13, 59-63

- Huertas, J. M. y Vilasaró, M. (1982) La premsa de barris a Barcelona 19391982. Barcelona: Servei de Premsa de la Diputació de Barcelona

- Lombardi, M. (1995). Ciudades con mensaje. En Entelequia, 5, 42-46. - López Cantos, F. (2004). El lugar de la ciudadanía. El Tercer Sector Audiovisual (TSA). En la web del Seminario de Estudios de la Comunidad y la Comunicación, Departamento de Ciencias da Comunicación, USC) Recuperada el 5
- Espinet, F. (1993). La gènesi de la so- de mayo de 2010 de http://www.usc.es/ xorna/SECCOM/textos/el lug cid.pdf

- López, O.; P. Vivas; J. Rojas; L. Farré, V. Santoro y T. Vidal (2006) Paseando por la ciberciudad: tecnología y nuevos espacios urbanos. Barcelona: Editorial UOC.

- Martín-Barbero, J. (1987). De los medios a las mediaciones. Comunicación, cultura y hegemonía. Barcelona: Gustavo Gili.

- Martín-Barbero, J. (1994). Mediaciones urbanas y nuevos escenarios de la comunicación. Caracas: Fundarte/ Ateneo de Caracas

- Mattelart, A. (2007). Historia de la sociedad de la información. Barcelona: Paidós.

- Mayugo, C. (2005). La audiovisibilidad, territorio ciudadano para ejercer el derecho a la comunicación. En Martínez, M. (Ed.). El Tercer Sector y el Audiovisual (pp. 39-48). Santiago de Compostela: Foro da Cidadanía e da Comunicación

- Miralles, A. M. (2001). Comunicación para el desarrollo urbano. PCLA-Pensamiento Comunicacional Latinoamericano, vol.3, 1 . Recuperada el 5 de mayo de 2010 de http://www2.metodista.br/ unesco/PCLA/revista9/documentos $\underline{9-1 . h t m}$

- Miralles, A.M. (2002). Periodismo, opinión pública y agenda ciudadana. Bogotá: Norma

- Moix, Ll. (1994). La ciudad de los arquitectos. Barcelona: Anagrama

- Reguillo, R. (1996). La construcción simbólica de la ciudad: sociedad, desastre y comunicación. Guadalajara, México: ITESO.

- Reguillo, R. (1997). Ciudad y comunicación. Densidades, ejes y niveles. Diálogos de FELAFACS, 47, 33-42.

- Sánchez, F. E. (1997). Cidade espetáculo. Política, planejamento e city marketing. Curitiba, Paraná (Brasil): Palabra.

- Sierra, F. (2006). Políticas de comunicación y educación: crítica y desarrollo de la sociedad del conocimiento. Barcelona: Editorial Gedisa.

- Silva, A. (1988). El territorio: una noción urbana. Signo y Pensamiento, Vol.7., 12, 81-91 


\begin{tabular}{|c|c|c|}
\hline & $>$ Referencias Bibliográficas & \\
\hline $\begin{array}{l}\text { - Traquina, N. y Mesquita, M. (2003) } \\
\text { Jornalismo Civico. Lisboa: Livros }\end{array}$ & $\begin{array}{l}\text { y competitividad. En Investigaciones } \\
\text { Regionales, } 1,139-161 .\end{array}$ & 723-738. \\
\hline $\begin{array}{l}\text { Horizonte. } \\
\text { - Trullén, J., Lladós, J. y Boix, R. (2002). } \\
\text { Economía del conocimiento, ciudad }\end{array}$ & $\begin{array}{l}\text { - Vivas, P., Ribera-Fumaz, R., López, } \\
\text { Ó. y Pellicer, I. (2008). Barcelona: una } \\
\text { ciberciudad en tránsito. En } A C E, 6 \text {, }\end{array}$ & $\begin{array}{l}\text { - Winocur, R. (2002). Ciudadanos } \\
\text { mediáticos. Barcelona: Gedisa. }\end{array}$ \\
\hline
\end{tabular}

\section{NOTAS BIOGRÁFICAS}

Anna Clua es profesora en la UOC, doctora en Ciencias de la Información por la UAB y especialista en Teorías de la Comunicación, realizando estancias en Dinamarca y Reino Unido. Ha desarrollado estudios sobre Ciudad y comunicación, acceso y uso social de las TIC o medios comunitarios.

Contacto: acluai@uoc.edu 